\title{
Erratum to: Preservation of positional identity in fetus-derived neural stem (NS) cells from different mouse central nervous system compartments
}

\author{
Marco Onorati ${ }^{1} \cdot$ Maurizio Binetti $^{1} \cdot$ Luciano Conti $^{1} \cdot$ Stefano Camnasio $^{1}$. \\ Giovanna Calabrese $^{2}$ - Ilaria Albieri ${ }^{2} \cdot$ Francesca Di Febo $^{3} \cdot$ Mauro Toselli $^{3}$. \\ Gerardo Biella $^{3} \cdot$ Ben Martynoga $^{4} \cdot$ Francois Guillemot $^{4} \cdot$ G. Giacomo Consalez ${ }^{2}$. \\ Elena Cattaneo ${ }^{1}$
}

Published online: 12 July 2016

(C) Springer International Publishing 2016

\section{Erratum to: Cell Mol Life Sci (2011) 68:1769-1783 DOI 10.1007/s00018-010-0548-7}

In the original publication the Fig. 2a showed an RT-PCR analysis of a panel of markers expressed by 9 lines of fetusderived NS and embryonic stem cells. The lane for Hoxb4 gene expression contained a white bar separating the positive control $(+\mathrm{CTRL})$. The band in the +CTRL was actually a duplication of the NS12SC band. In fact, the general mouse fetal brain, used as +CTRL for all the other markers, did not express the spinal cord marker Hoxb4. In the new version of the Fig. 2 we removed the duplicated + CTRL band for Hoxb4.

Then, in Fig. 5c-d, there was a redundant duplication of the PCR for $H b 9, \operatorname{lr} x 3$ and $N k x 2-2$, shown at first for spinal cord NS12SC in Fig. 5c, and then compared side-by-side with striatal NS12ST in Fig. 5d. In the new version, duplicated PCR analysis on NS12SC cells was removed from Fig. $5 \mathrm{~d}$ and is referred to the data shown in Fig. $5 \mathrm{c}$ in the new figure legend.

The online version of the original article can be found under doi:10.1007/s00018-010-0548-7.

Elena Cattaneo

elena.cattaneo@unimi.it

1 Department of Pharmacological Sciences and Center for Stem Cell Research, Universitá degli Studi di Milano, Via Balzaretti 9, 20133 Milan, Italy

2 San Raffaele Scientific Institute and Universitá Vita-Salute San Raffaele, Milan, Italy

3 Department of Cellular and Molecular Physiological and Pharmacological Sciences, University of Pavia, Pavia, Italy

4 National Institute for Medical Research, London, UK 
Fig. 2 Analysis of the in vitro positional identity in NS cell lines. a RT-PCR analysis of a subset of R-C markers shows that fetus-derived NS cells possess a region-specific transcription factor pattern: Foxgl is expressed in telencephalic NS lines, Otx2 and Six3 in anterior CNSderived NS cells, and Hoxb4 and Hoxb9 in NS12SC cells. Mouse fetal brain was used as positive control $(+C T R L)$; - RT was the negative control $\mathbf{b}$, c RT-qPCR analysis showing the expression levels of two relevant R-C markers, Foxg1 (b) and Hoxb4 (c). Data are expressed as percentages (100\% was assigned to fetal CNS-E13 brain and spinal cord). d RT-PCR analysis of some indicative $\mathrm{D}-\mathrm{V}$ markers shows constitutive expression of important ventral markers, including Meis2 and Ascl1. Mouse fetal brain was used as positive control $(+C T R L)$; - RT was the negative control. e, f RT-qPCR analysis showing the expression levels of two relevant D-V markers, $\operatorname{Pax} 7$ (e) and Ascll (f). Data are expressed as percentages (100\% was assigned to fetal CNS). ${ }^{*} p<0.05, * * p<0.01$, $* * * p<0.001$, as calculated by ANOVA using the TukeyKramer post-test
A
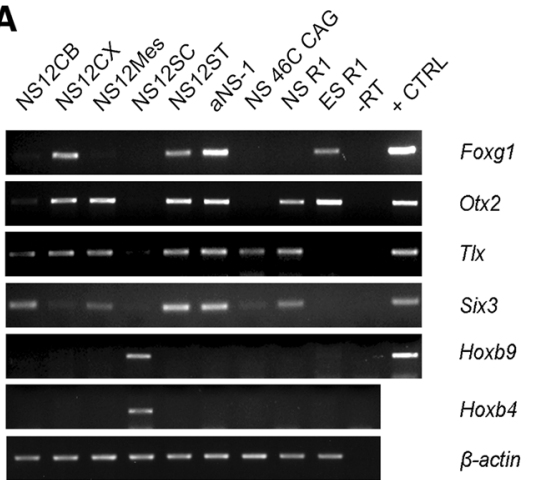

C

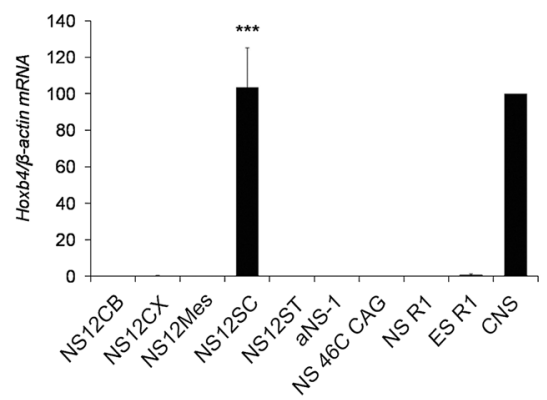

E

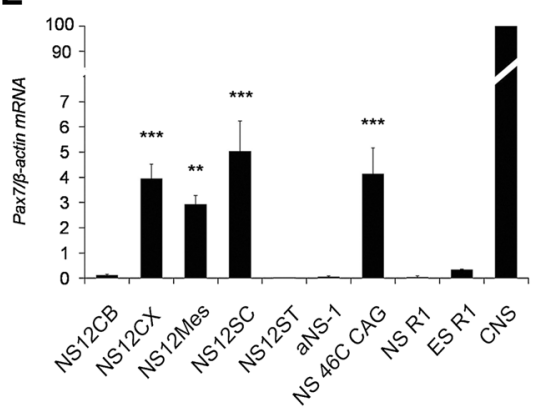

B

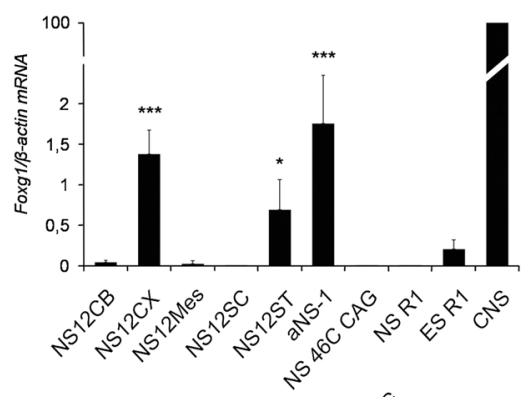

D
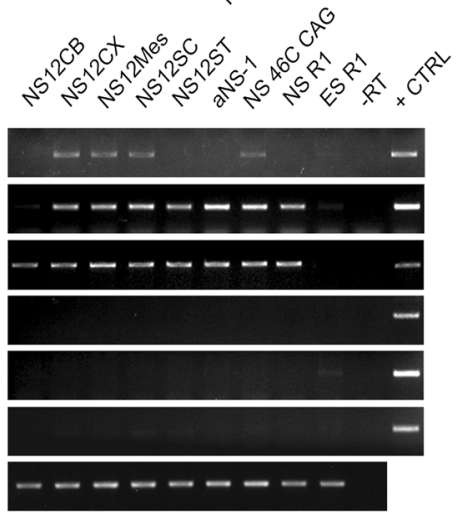

Pax7

Meis2

Ascl1

Nkx2-1

Gsx2

Shh

$\beta$-actin

F

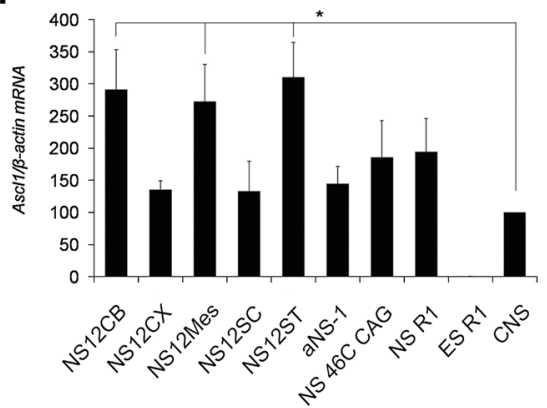


Fig. 5 Neuronal differentiation of NS12SC cells. a After 23 days of differentiation in vitro, NS12SC cells gave rise to $\beta$ III-tubulin-, MAP2-, GFAP-, and O4-positive cells. A representative synapsin-positive cell is shown after 21 days of differentiation $(x 4$, magnified after acquisition). b At the end of differentiation, $50.5 \pm 3.9 \%$ of cells were immunopositive for $\beta$ III-tubulin, $57.7 \pm 10.3 \%$ for MAP2, $7.5 \pm 2.8 \%$ for GFAP, and $1.5 \pm 0.7 \%$ for $\mathrm{O} 4$ ( $n=1068$ cells for $\beta$ III-tubulin; $n=1184$ cells for MAP2 and GFAP; $n=1645$ cells for O4) (columns represent averages, error bars standard deviations). c Gene expression analysis by RT-PCR on NS12SC cells in proliferation $(P)$ and after 15 days differentiation in the absence $(D 15-M)$ or in the presence $(D 15+M)$ of morphogens. d Spinal gene markers analyzed in $\mathbf{c}$ are not detected in NS12ST cells.

Mouse fetal brain was used as positive control $(+C T R L) ;-R T$ was the negative control

\section{A}
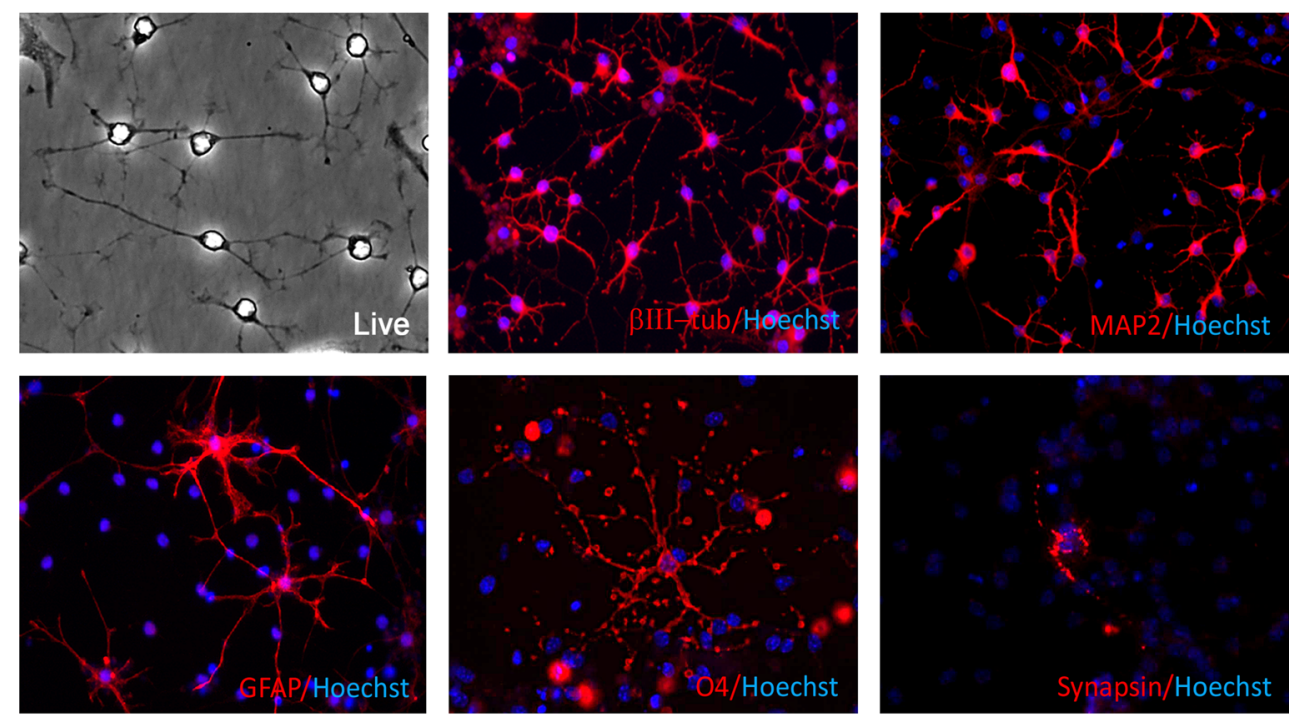

c

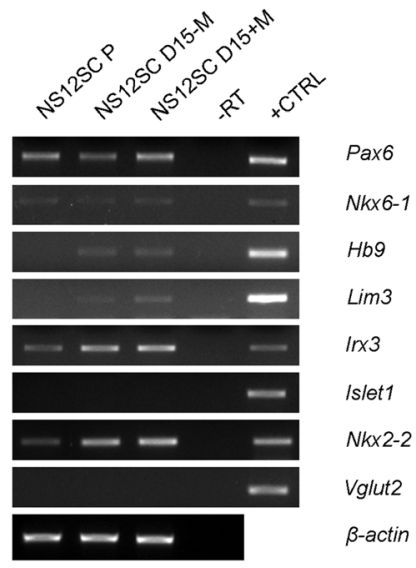

D

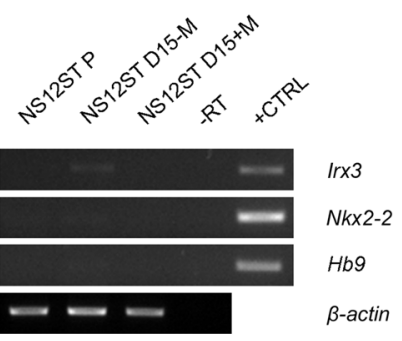

